\title{
Superfluid phases of the three-species fermion gas
}

\author{
Paulo F. Bedaque \\ Lawrence-Berkeley Laboratory, Berkeley, CA 94720, USA and \\ University of Maryland, College Park MD, 20742 USA \\ José P. D'Incao \\ Department of Physics, Kansas State University, Manhattan, KS 66506, USA
}

\begin{abstract}
We discuss the zero temperature phase diagram of a dilute gas with three fermionic species. We make use of solvable limits to conjecture the behavior of the system in the "unitary" regions. The physics of the Thomas-Efimov effect plays a role in these considerations. We find a rich phase diagram with superfluid, gapless superfluid and inhomogeneous phases with different symmetry breaking patterns. We then discuss one particular possible experimental implementation in a system of ${ }^{6} \mathrm{Li}$ atoms and the possible phases arising in this system as an external magnetic field is varied across three overlaping Feshbach resonances. We also suggest how to experimentally distinguish the different phases.
\end{abstract}

Cold atomic gases provides are great laboratory for many-body physics. This is due to the variety of atomic isotopes that can be trapped and the tunability of the atomic interactions. Another point of interest is the "universality" of some of their properties: since the detailed shape of the interatomic potential is not probed in dilute systems, many different kinds of interactions can be approximated by a contact potential and different systems present analogous characteristics.

Our goal in this letter is to explore the phase diagram of a dilute ultracold gas composed of three fermionic species [1]. It can be considered a generalization of the well studied two fermionic species gas and brings about some new features like competition among interactions and the consequences of the Efimov efect [2] Let us first summarize the main properties of the two-fermion case as they will be useful in the more involved three-fermion gas. There are three relevant distance scales in this system: $r_{0}$ measures the range of the interatomic forces, $a$ is the s-wave scattering length and $1 / n^{1 / 3}$ the typical interatomic distance ( $n$ is the particle density). We will consider only the cases where $r_{0} \ll a, 1 / n^{1 / 3}$ since only in this case the results have some degree of universality. The cases where $n|a|^{3} \ll 1$, where an expansion in powers of $n|a|^{3}$ is possible, are more easily analyzed. In the regime $n a^{3} \ll 1, a>0$ the atoms bind into diatomic molecules much smaller than the separation between them while interacting weakly with each other. These molecules then condense to form a weakly coupled superfluid. If $a<0$, though, there are no molecules. Still, the atoms form Cooper pairs and the ground state of the system is a BCS superfluid. In the intermediate regime $-1 \lesssim 1 / n a^{3} \lesssim 1$, the so-called "unitary" region, the absence of a small parameter complicates the theoretical analysis but one can still infer some of its qualitative properties. Notice that this region is scaled down (in energy) version of the dilute neutron gas, exemplifying the universality pointed out above. In both the BEC limit $1 / n a^{3} \gg 1$ and the BCS limit $1 / n a^{3} \ll-1$ the condensation of molecules or
Cooper pairs breaks the wave function phase symmetry. That is, if we denote by $\psi_{1}\left(\psi_{2}\right)$, the annihilation operator for the atom of type 1 (2), the symmetry of the hamiltionian $\psi_{1,2} \rightarrow e^{i \alpha} \psi_{1,2}$ is not a symmetry of the ground state due to the non-vanishing value of $\left\langle\psi_{1} \psi_{2}\right\rangle$. Since the symmetry breaking pattern is the same on both sides of the "unitary" region there is not reason to expect a qualitative change (a phase transition) in between the BEC and the BCS limits. This picture is vindicated both by experimental [3] and numerical [4] results.

Similar arguments can be made in the three-fermion case of interest here. There are two main new ingredients absent in the two-fermion case. The first is the competition between the pairing/binding among different atom pairs. The second is related to three-body correlations. Ordinarily, two-body correlations dominate the physics of dilute systems but that may not be true in the regime $r_{0} / a \ll 1$. Indeed the Thomas effect [5] (the collapse of the three-body system as $r_{0} \rightarrow 0$ at fixed $a)$ and the Efimov effect [2] (the accumulation of threebody bound states at threshold as $a \rightarrow \infty$ at fixed $r_{0}$ ) are consequences of the strong attraction among three resonating particles. These two closely related phenomena [6] can occur only if the three particles in the system can overlap in space, which is forbidden in the case of only two fermion species. The sensitivity to the physics of three overlapping particles brings out a dependence of the low energy processes in a new parameter $[2,7]$ unrelated to two-body scattering which, in the language of Effective Field Theories, is the value of a contact threebody force [8]. Currently, the experimental observation of the Efimov effect is a subject of great interest due to the fundamental aspects it represents for the three-body physics in ultracold quantum gases. Recently an analysis of the three-body recombination [9] in Bose-Einstein condensates have shown evidence of this intriguing effect [10].

At zero temperature, with equal densities of the three species, the phase diagram of the system is a function 


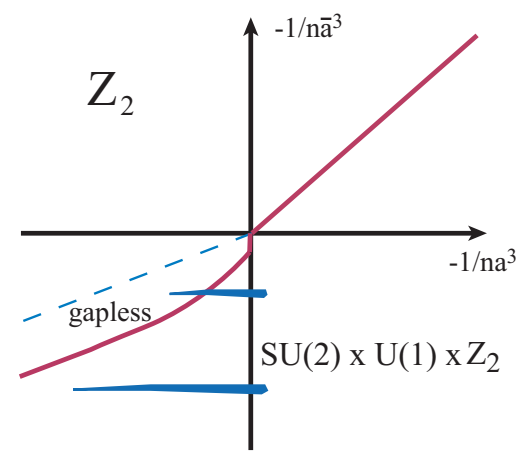

FIG. 1: Conjectured phase diagram. The horizontal bands (blue online) show the narrow areas of atom-molecule separation. The solid (red online) represent the phase boundary between the $\mathbb{Z}_{2}$ and $S U(2) \times U(1) \mathbb{Z}_{2}$ phases. The dashed line is where a Fermi surface appears (Lifshitz transition).

of four dimensionless variables. The dependence on the three-body parameter is limited so we keep it fixed. The remaining three parameters can be taken to be $n a_{i j}^{3},\left(a_{i j}\right.$ is the scattering length between particles $i$ and $j$ and $n$ the density of each atomic species). We will initially restrict our discussion to the slightly idealized situation where two of the scattering lengths are equal, thus assuming $a_{12}=a_{23}=a, a_{31}=\bar{a}$. Our strategy to map the zero temperature phase diagram as a function of $a$ and $\bar{a}$ will be to understand the system in the soluble limits of the phase diagram where $n|a|^{3}, n|\bar{a}|^{3} \ll 1$ and make educated guesses about how to interpolate between these limits. This is the same successful strategy discussed above for the two-fermion case.

The system can be described by the hamiltonian

$$
\begin{aligned}
\mathcal{H} & =-\sum_{i=1}^{3} \psi_{i}^{\dagger} \frac{\nabla^{2}}{2 M} \psi_{i}+\bar{g}^{2}\left|\psi_{1}\right|^{2}\left|\psi_{3}\right|^{2} \\
& +g^{2}\left(\left|\psi_{1}\right|^{2}\left|\psi_{2}\right|^{2}+\left|\psi_{2}\right|^{2}\left|\psi_{3}\right|^{2}\right)+G\left|\psi_{1}\right|^{2}\left|\psi_{2}\right|^{2}\left|\psi_{3}\right|^{2}
\end{aligned}
$$

The couplings $g, \bar{g}$ and $G$ depend on a cutoff and are determined respectively by $a, \bar{a}$ and, in the case of $G$, on a three-body observable like one of the atom-dimer scattering lengths for some value of $a, \bar{a}$. The hamiltonian in eq. (1) has a $S U_{13}(2) \times U_{2}(1) \times U_{123}(1)$ symmetry, where the first factor acts only on atoms of type " 1 " and " 3 ", the second on atoms of type "2" only and the last is a common phase change in all atom fields.

Let us first consider the case where both scattering lengths are positive and $n a^{3}, n \bar{a}^{3} \ll 1$, located in the third quadrant in Fig. 1. There all three pairs can form bound states of energy $B=1 / M a^{2}$ or $\bar{B}=1 / M \bar{a}^{2}$ and whose spatial extent is much smaller than the interparticle distance. If we denote the number of bound states between particles $i$ and $j$ by $n_{i j}$ and the number of unbound atoms of type $i$ by $n_{i}$ we have that, to leading order in $n a^{3}$ and $n \bar{a}^{3}$, the energy of the system is given by

$\mathcal{E}=\frac{3\left(6 \pi^{2}\right)^{2 / 3}}{10 M}\left(n_{1}^{5 / 3}+n_{2}^{5 / 3}+n_{3}^{5 / 3}\right)-B\left(n_{12}+n_{23}\right)-\bar{B} n_{13}$,

which is just the sum of the molecule binding energies and the energy of the Fermi gas of unbound atoms. We disregarded the interaction between unbound atom-dimer, unbound atom-unbound atom and dimer-dimer as these contribution are, as long as their respective scattering lengths are of the order of $\sim a, \bar{a}$, suppressed by powers of $n a^{3}, n \bar{a}^{3} \ll 1$. As we will see later the atom-dimer scattering length may diverge for some very particular values of the parameters as a consequence of the three-body dynamics and will be necessary to amend this analysis. We find the ground state by minimizing eq. (2) with the restriction that the total number of atoms of each species is $n\left(n_{1}+n_{12}+n_{31}=n\right.$ and similarly for the other species). We find three different cases:

$$
\begin{aligned}
\bar{B} & <2 B: n_{1}=n_{2}=n_{3}=0, n_{12}=n_{23}=n_{31}=n / 2 \\
2 B & <\bar{B}<2 B+\frac{\left(6 \pi^{2} n\right)^{2 / 3}}{M}: n_{1}=n_{3}=0, n_{2}=\frac{(\bar{B}-2 B)^{3 / 2}}{6 \pi^{2}}, \\
\bar{B} & >2 B+\frac{\left(6 \pi^{2} n\right)^{2 / 3}}{M}: n_{1}=n_{3}=0, n_{2}=n .
\end{aligned}
$$

This result is physically clear. In the first case, it is favorable for all atoms to form bound states, and $n / 2$ dimers are formed between each species pair. All dimers species condense leading to a non-zero value of the ground state expectation value $\left\langle\psi_{i} \psi_{j}\right\rangle$ for all $i, j$. This condensate breaks the symmetry from $S U_{13}(2) \times U_{2}(1) \times U_{123}(1)$ down to $\mathbb{Z}_{2}$, corresponding to a common sign flip on all atom fields. If $\bar{B}$ increases (i.e., $\bar{a}$ decreases) beyond $\bar{B}>2 B$ it is favorable to break some of the " 12 " and " 23 " dimers in order to increase the number of " 31 " dimers, leaving some "2" atoms unbound. The condensates $\left\langle\psi_{1} \psi_{2}\right\rangle$ and $\left\langle\psi_{2} \psi_{3}\right\rangle$ will decrease in value but the symmetry breaking pattern will remain the same. The formation of a Fermi surface for the "2" atoms indicates a topological (Lifshitz) phase transition (denoted by the dashed line in fig. (1)). As we keep increasing $\bar{B}$ (decreasing $\bar{a}$ ) there comes a point where all "2" atoms are unbound and only "13" dimers can be found. At this point $\left\langle\psi_{1} \psi_{2}\right\rangle=\left\langle\psi_{2} \psi_{3}\right\rangle=0$ and the symmetry breaking pattern becomes $S U_{13}(2) \times U_{2}(1) \times U_{123}(1) \rightarrow$ $S U_{13}(2) \times U_{2}(1) \times \mathbb{Z}_{2}$. The change in symmetry of the ground state indicates the presence of a phase transition, marked by the full line in the third quadrant of fig. (1).

We now consider the opposite side of the phase diagram, i.e., the region where $a, \bar{a}<0$, and $n|a|^{3}, n|\bar{a}|^{3} \ll 1$ (first quadrant in fig. 1). In this region there are no twobody bound states, however, symmetries can be broken by the condensation of Cooper pairs. The most general s-wave condensate can be written as

$$
\left\langle\psi_{i} \psi_{j}\right\rangle=\epsilon_{i j k} \Delta^{k}
$$


where $\overrightarrow{\boldsymbol{\Delta}}=\left(\Delta_{23}, \Delta_{31}, \Delta_{12}\right)$ is a complex vector describing the fermionic gaps. In the regime considered the BCS theory (mean field) can be used. Using the methods of reference [12] we find the thermodynamical potential in this approximation to be

$$
\begin{aligned}
\mathcal{E}-\mu \mathcal{N}_{i} & =\frac{1}{2} \int \frac{d^{3} k}{(2 \pi)^{3}}\left(3 \epsilon(k)-|\epsilon(k)|-2 \sqrt{\epsilon^{2}(k)+|\boldsymbol{\Delta}|^{2}}\right. \\
& \left.+\frac{2 M}{k^{2}}|\boldsymbol{\Delta}|^{2}\right)-M \frac{\left|\Delta_{12}\right|^{2}+\left|\Delta_{23}\right|^{2}}{4 \pi a}-M \frac{\left|\Delta_{31}\right|^{2}}{4 \pi \bar{a}}(4)
\end{aligned}
$$

where $\epsilon(k)=k^{2} / 2 M-\mu$ and $\mu$ is the chemical potential. In eq. (4) we traded the couplings constants by the scattering lengths. In general, in order to maintain equal densities for the three species, different values for the chemical potentials for the three species are required. At low density though, those differences (of the order of $\Delta^{2} / k_{F}$, where $\Delta$ is the gap and $k_{F}$ the Fermi momentum) are suppressed by the exponentially small size of the gap and will be neglected here. The ground state is found by minimizing eq. (4). The first term in eq. (4) is a function only of the combination $|\boldsymbol{\Delta}|^{2}$ which is a $S U_{123}(3)$ invariant. The remaining terms don't have this symmetry and, consequently, tip the balance in favor of one direction in $\boldsymbol{\Delta}^{k}$ space. The location of the minimum then depends on the competition between the strength of the interaction between the atoms, that is, on relative sizes of $a$ and $\bar{a}$. If $|\bar{a}|>|a|$, the energy is minimized by having $\Delta_{13} \neq 0$ and $\Delta_{12}=\Delta_{23}=0$. The atoms of type "1" and " 3 " pair up with each other while the atoms of type "2" remain unpaired. The symmetry breaking pattern is $S U_{13}(2) \times U_{2}(1) \times U_{123}(1) \rightarrow S U_{13}(2) \times U_{2}(1) \times \mathbb{Z}_{2}$. In the opposite case, $|\bar{a}|<|a|$, the energetically favored state has $\Delta_{13}=0$ and $\left|\Delta_{12}\right|^{2}+\left|\Delta_{23}\right|^{2} \neq 0$. It is equally favorable for $\Delta_{12}$ or $\Delta_{23}$, or a combination of them to be non-zero, due to the $S U_{13}(2)$ symmetry of the hamiltonian. The symmetry breaking pattern is $S U_{13}(2) \times U_{2}(1) \times U_{123}(1) \rightarrow \mathbb{Z}_{2}$. In this phase atoms of type " 1 " and " 3 " do not pair up with each other but pair up with atoms of type " 2 ". As a consequence of the different pairing patterns we conclude that there must be a phase transition along the line $a=\bar{a}$ in the first quadrant. Both phases are superfluid since both contain Cooper pairs but the $S U_{13}(2) \times U_{2}(1) \times \mathbb{Z}_{2}$ phase also has metal-like properties on account of the unpaired atoms of type "2".

Let us consider now the second quadrant in (Fig. 1), approaching it from the third quadrant by moving along the vertical direction. As we saw, in the third quadrant just below the horizontal axis all atoms are bound. As we cross the horizontal axis, atoms of type "1" and " 3 " are no longer bound but can still form Cooper pairs. In fact, the transition between bound atoms and Cooper pairs does not change the symmetries of the ground state (that remains $\mathbb{Z}_{2}$ ) and no phase transition is expected. This is perfectly analogous to the BEC/BCS transition discussed of the two-fermion system. Finally, let us consider the fourth quadrant. There is also a smooth connection between third and fourth quadrants as $a$ flips sign. In the third quadrant, just to the left of the vertical axis, atoms "2" are attracted to the other ones, but all atoms "1" and " 3 " are already bound and not available for binding with " 2 " and the attraction to atoms " 2 " is not strong enough to force a rearrangement of the binding. As we move horizontally towards the right and cross the vertical axis, the interaction of atoms of type "2" with atoms " 1 " and " 3 " becomes weaker and does not even support a bound state. Consequently, no qualitative change occurs and no phase transition is expected. The symmetry breaking pattern remains the same and no phase transition is expected.

Up to now we have taken into account only two-body correlations - the existence of two-body bound states and/or Cooper pairs - to discuss the ground state of the system. This is reasonable in dilute systems with short range interactions as the probability of having more particles at the same point in space is small. Our previous discussion receives corrections proportional to $n|a|^{3}, n|\bar{a}|^{3} \ll 1$ and is, consequently, robust. This argument however, implicitly assumes that parameters describing the three-body physics, for instance, the scattering length between an atom and a molecule, are of the same order as $a$ or $\bar{a}$. The presence of a three-body scale ,however, may invalidate the argument. This is indeed what happens for some specific values of the parameters. It is known that the atom-dimer scattering length becomes arbitrarily large for specific values of the parameters. When the atom-dimer interaction is strong and repulsive the possibility of spatial separation between atoms and molecules arises. Free atoms and molecules appear simultaneously only in the third quadrant of the phase diagram and only the atom of type " 2 " is found unbound among molecules of type " 13 ". The criterion for the (linear) stability against atom/molecule separation in a boson-fermion (atom-molecule) mixture is [13]

$$
\frac{\left(6 \pi^{2}\right)^{5 / 3}}{9 \pi} \frac{a_{13,13}}{n_{2}^{1 / 3}}>9 \pi^{2} a_{13,2}^{2},
$$

where $a_{13,13} \approx 0.6 \bar{a}[14]$ is the scattering length between two "13" dimers and, for $\bar{a}<a$ we have [2]

$$
a_{13,2}=\bar{a}\left(A+B \tan \left(s_{0} \log \frac{\bar{a}}{r_{0}}+\Phi\right)\right),
$$

where $A, B$ and $s_{0} \approx 1.006$ are numerical constants. Unfortunately, $\Phi$ is a function of the three-body force $G$ and thus can only be determined with the knowledge of a three-body observable. For $a<\bar{a}$, there is no enhancement and $a_{13,2} \sim \bar{a}$. Notice that a such divergence of the atom-dimer scattering length (the poles in Eq. (6)) only occur for fermionic gases if there is, at least, three different species. In other cases the Pauli principle forbids the atoms to overlap and limits the strength of the 
interaction among the three particles. For $\bar{a}$ near one of the poles of Eq. (6) the stability condition given by Eq. (5) is violated and atoms of type "2" separate from the "13" molecules. The experimental observation of this phenomenon, as well as its repetition for different poles in $a_{13,2}$, would provide a striking confirmation of the Efimov effect. The bands where this instability occurs are repeated every time $\bar{a}$ is increased by a factor $e^{\pi / s_{0}} \approx 22.7$. We have indicated two of these bands in our phase diagram in Fig. 1. Their fate as we cross the vertical axis is unclear, i.e., they may or may not extend into the fourth quadrant. The precise position where these bands occur when we approach the unitary regime $\left(-1 / n|a|^{3} \ll 1\right)$ varies for each systems as it depends on three-body information encapsulated in $G$. It is hard to determine whether this repetition continues to very small values of $-1 / n|a|^{3}$ and we then enter the unitary regime where our methods fail.

Our considerations can be easily extended for the case where all three atom-atom scattering lengths are different. Here we will consider the phases arising in a concrete possible realization of the three-fermion gas. It was predicted in Ref. [11] the existence of three Feshbach resonances between atoms in the lowest three hyperfine states of ${ }^{6} \mathrm{Li}$ for values of the magnetic field $\mathbf{B}$ in the range of $60 \mathrm{mT}<\mathbf{B}<100 \mathrm{mT}$. In this case, all scattering lengths are positive for small fields and negative for large fields. The resonances occur around $\mathbf{B} \approx 69$ $\mathrm{mT}$ ("13"), $\mathbf{B} \approx 81.1 \mathrm{mT}$ and ("12") and $\mathbf{B} \approx 83.4 \mathrm{mT}$ ("23"), where the respective scattering length diverges and flip sign. For the largest $\mathbf{B}$ in this range all $a_{i j}$ are negative, the ground state of the system is an analogue of the phase in the first quadrant in fig. (1) and the condensate is driven by the largest scattering length in absolute value. A $\left\langle\psi_{1} \psi_{2}\right\rangle$ condensate forms which breaks the $U_{1}(1) \times U_{2}(1) \times U_{3}(1)$ symmetry of the hamiltonian down to $U_{1-2}(1) \times U_{3}(1) \times \mathbb{Z}_{2}\left(U_{1-2}(1)\right.$ corresponds to an equal but opposite phase rotation of the atoms " 1 " and "2"). As the field is lowered below $\mathbf{B}=81.1 \mathrm{mT}, a_{12}$ and $a_{23}$ flip sign but that initially does not change the phase of the system. Atoms " 1 " and " 2 " that formed a Cooper pair at large $\mathbf{B}$ now form a bound state. Lowering $B$ further still, at some point the binding energies for the pairs " 12 " and " 23 " are close to each other and large enough to dominate the energetics of the system. At this point, type " 12 " and " 23 " molecules form and condense. The creation of condensates $\left\langle\psi_{1} \psi_{2}\right\rangle$ and $\left\langle\psi_{2} \psi_{3}\right\rangle$ break the symmetry down to $U_{1}(1) \times U_{2}(1) \times U_{3}(1) \rightarrow \mathbb{Z}_{2}$, indicating the existence of a different phase from the one at high values of $\mathbf{B}$. This is the analogue of the phase transition in the third quadrant of fig. (1). As we mentioned above, this picture may be modified by the atom-molecule separation for certain values of $\mathbf{B}$ below the " 12 " resonance, but we cannot predict for which value of $\mathbf{B}$ this will occur due to our ignorance of the parameter $\Phi$ (or $G$ ). The phase separation would be observed in practice if one would be able to scan $a_{12}$ through a factor of $e^{\pi / s_{0}} \approx 22.7$ in order to be sure to cross one of instability bands. A measurement of three-body observables involving these three states of ${ }^{6} \mathrm{Li}$ would determine $G$ (and $\Phi$ ) and would allow us to make a more quantitative prediction.

We close by pointing out that the two different superfluid phases can be distinguished by the properties of their vortices. In the three ${ }^{6} \mathrm{Li}$ system, for instance, and at high fields, atoms of type " 3 " are unpaired and should not participate in in the formation of superfluid vortices. For lower fields, below the point where the phase transition occur all atom types can only rotate by the formation of vortices. The creation of these vortices by means of probes coupling to only one atom type is a direct way of exploring the phase diagram we have discussed.

[1] A similar system was considered in C. Honerkamp and W. Hofstetter, Phys. Rev. Lett. 92, 170403 (2004), C. Honerkamp and W. Hofstetter, Phys. Rev. B 70, 094521 (2004).

[2] V. Efimov, Sov. J. Nucl. Phys. 12589 (1971), V. Efimov, Sov. J. Nucl. Phys. 29546 (1979).

[3] K.M.Ohara et al., Science, 298, 2179 (2002); M.E. Gehm, Phys. Rev. A 68, 011401 (2003); C. A. Regal et al., Nature, 42447 (2003); J. Cubizolles et al., Phys. Rev. Lett. 91, 240401 (2003); K. E. Strecker et al., Phys. Rev. Lett. 92, 120401 (2004), Phys. Rev. Lett. 91, 080406 (2003); S. Jochim, Phys. Rev. Lett. 91, 240402 (2003), J. Kinast et al., Phys. Rev. Lett. 92, 150402 (2004), M. W. Zwierlein et al., Phys. Rev. Lett. 92, 120403 (2004), C. A. Regal et al., Phys. Rev. Lett. 92, 040403 (2004), C. Chin et al., Science 305, 1128 (2004), J. Kinast et al., Science, 301, 1296 (2005).

[4] J. Carlson et al., Phys. Rev. Lett. 91, 050401 (2003); G. E. Astrakharchik et al., Phys. Rev. Lett. 93, 200404 (2004); A. Bulgac et al., arXiv:cond-mat/0505374; D. Lee and T. Schafer, Phys. Rev. C 72, 024006 (2005).

[5] L. H. Thomas, Phys. Rev. 47 (1935) 903.

[6] For a nice review see E. Braaten and H. W. Hammer, arXiv:cond-mat/0410417.

[7] G. S. Danilov, Sov. Phys. JETP 13, 349 (1961).

[8] P. F. Bedaque, H. W. Hammer and U. van Kolck, Phys. Rev. Lett. 82, 463 (1999), P. F. Bedaque, H. W. Hammer and U. van Kolck, Nucl. Phys. A 676, 357 (2000), R. F. Mohr, R. J. Furnstahl, R. J. Perry, K. G. Wilson and H. W. Hammer, arXiv:nucl-th/0509076.

[9] P. O. Fedichev et al., Phys. Rev. Lett. 77, 2921 (1996); E. Nielsen and J. H. Macek, Phys. Rev. Lett. 83, 1566 (1999); B. D. Esry, C. H. Greene, and J. P. Burke, Phys. Rev. Lett. 83, 1751 (1999); P. F. Bedaque, Eric Braaten and and H.-W. Hammer, Phys. Rev. Lett. 85, 908 (2000); E. Braaten and H. W. Hammer, Phys. Rev. Lett. 87, 160407 (2001).

[10] T. Kraemer et al., arXiv:cond-mat/0512394.

[11] M. Bartenstein et al., Phys. Rev. Lett. 94, 103201 (2005).

[12] Lieb, Schultz and Mattis, Ann. of Phys., 16, 407 (1961).

[13] C. J. Pethick and H. Smith, Bose-Einstein Condensation in Dilute Gases, Cambridge University Press. 
[14] D.S. Petrov et al., Phys. Rev. Lett. 93, 090404 (2004). 